(C)2007 IEEE. Personal use of this material is permitted. However, permission to reprint/republish this material for advertising or promotional purposes or for creating new collective works for resale or redistribution to servers or lists, or to reuse any copyrighted component of this work in other works must be obtained from the IEEE 


\title{
State of the art review of the existing soft computing based approaches to trust and reputation computation
}

\author{
Farookh Khadeer Hussain, Elizabeth Chang and Omar Khadeer Hussain \\ Digital Ecosystems and Business Intelligence Institute \\ Curtin University of Technology Perth, Australia \\ E-mail: \{Farookh.Hussain,Elizabeth.Chang,Omar.Hussain\}@cbs.curtin.edu.au
}

\begin{abstract}
In this paper we present a state of the art review of the soft computing based approaches for trust and reputation computation. We divide the soft computing based approaches for trust and reputation computation into five different classes. Each of the five different classes is discussed in this paper.
\end{abstract}

\section{Introduction}

Lately, researchers are making use of soft computing methods like fuzzy logic and neural networks for trust modeling and reputation modelling. Notable researchers who have made use of soft computing methods in order to model trust and reputation are Sabater et al, Aringheri et al, Shanshan Song et al, Weihua Song et al, Carter and Ghorbani et al, Ramchurn et al etc.

In this paper we present an overview of the proposed and existing soft computing methods for trust and reputation computation. For the purpose of discussion we classify the existing trust management protocols into 5 broad classes, namely: (a) ReGreT, proposed by Sabater et al. This is discussed in Section 2; (b) Fuzzy Logic Based Approach to trust and reputation computation proposed by Shanshan Song et al. This is discussed in Section 3; (c) Fuzzy Logic Based Approach to trust and reputation computation proposed by Weihua Song et al. This is discussed in Section 4; (d) Fuzzy Logic Based Approach to trust and reputation computation proposed by Carter and Ghorbani et al. This is discussed in Section 5; and (e) Fuzzy Logic Based Approach to trust and reputation computation proposed by Ramchurn et al. This is discussed in Section 6.

\section{ReGreT}

Sabater et al $[1,2,3]$ propose a fuzzy logic based approach for modelling reputation, termed as ReGreT. ReGreT is applicable where agents are organized as and work in groups. In ReGreT a given agent can have three types of reputation, namely individual dimension, social dimension and ontological dimension.

The individual dimension of reputation of an agent (say A) is used to reflect the individual impression that another agent (say B) has with it, in a given context and at a given point in time. The individual dimension of reputation can take just two values namely -1 and 1 .

The social dimension of reputation of an agent (say A) is computed by determining the reputation of the group to which agent A belongs. The main idea is that an agent inherits by default the reputation of the group to which it belongs. An agent (say B) determines the social dimension of reputation of an agent (say A) as a weighted average of four factors namely; (a) the individual dimension of reputation of agent $\mathrm{A}$ as held by agent $\mathrm{B}(\mathrm{b})$ the individual dimension of reputation of the group members of Agent A as held by Agent B (c) the individual dimension of reputation of Agent $A$ as held by the group members of Agent B and (d) the individual dimension of reputation of the group members of Agent $\mathrm{A}$ as held by the group members of Agent B.

Agent B makes use of fuzzy logic rules in order to ensure that the recommendations about Agent A are not biased or incorrect. Additionally, fuzzy logic based rules are made use of to compute the aggregated reputation of all the group members of both Agent A and Agent B. It should be noted here that along the individual and social dimensions, reputation is always 
linked to a single context. In order to model the dynamic nature of reputation, the reputation value is decayed, as a function of time. The ontological dimension of reputation combines the social and individual dimension of reputation of an agent in different contexts into a single numerical value.

\section{Fuzzy Logic Based Approach to trust and reputation computation proposed by Shanshan Song et al}

Shanshan Song et al [4-6] propose a fuzzy logicbased method for making trust-based decisions regarding interactions in Grid Networks. In their proposed method, a given site (say site A) makes a trust-based decision about another site (site B) based on the aggregated value of two factors, namely the self defense capability of site B and the reputation of site B.

Site A uses fuzzy rules to aggregate the aforesaid two factors in order to determine the trust level of site B.

Each site determines its own self-defense capability as a function of its capabilities in four factors, namely: (a) the effectiveness of its intrusion defense system; (b) its effectiveness in warding off viruses (anti-virus capability); (c) the effectiveness of its firewall; and (d) its secure job execution capabilities. Fuzzy rules are made use of to combine the above four factors to determine the self-defense capability of a given site, which is available upon request to other sites.

Additionally, each site in the network (say A) determines the reputation of other site(s) in the network (say B) as a function of four different factors, namely (a) its prior job execution success rate; (b) its job turnaround time; (c) its job slowdown ratio; and (d) its cumulative site utilization. Site A makes use of fuzzy rules to aggregate the above four factors to determine the reputation of Site $B$.

\section{Neural Network Based Approach to trust and reputation computation proposed by Weihua Song et al}

Weihua Song et al [7-8] propose a neural networkbased approach to determine the global reputation value of agents in P2P Networks. They propose a 'master-slave trust management structure' in which there is a single master agent who is responsible for keeping track of the global reputation value of each user in the network.

On the other hand, there are multiple slave agents who keep track of the reputation of a given user in a given context only. Each of the slave agents observe and evaluate the behaviour of the users in a given context only and aggregate the trust value(s) of the users in that context only to determine its reputation value. The master agent is responsible for assigning users to different slave agents, depending on the context in which the agents interact.

The master agents make use of a neural network to aggregate the multiple local reputations of a given user (in different contexts) which are stored with the slave agents to determine the global reputation of that user. The neural network is developed and trained by the master agent till the global reputation value of the user as determined by the neural network is close to (with in acceptable limits) the global reputation value determined by the HISTOS algorithm for computing reputation proposed by Zacharia et al [9].

\section{Fuzzy Logic Based Approach to trust and reputation computation proposed by Carter and Ghorbani et al}

Carter and Ghorbani et al [10, 11, and 12] propose a role-based method for modelling trust and reputation that makes use of fuzzy logic rules to aggregate the reputation of an agent in different roles to determine the global reputation of an agent. They propose the applicability of their proposed method in two domains namely a Multi-agent System [10,11] and an Information Sharing Society [12].

They contend that an agent in a multi-agent System (MAS) can play three roles, namely: as an assistant (an agent say A can aid or help its owner in carrying out its activities), as a service provider (an agent can provide services to other agents in a multi-agent system) and as a citizen (an agent can be a denizen of a multi-agent system). They develop metrics to quantify and express the extent to which an agent (say A) has fulfilled each of the above three roles (termed as the reputation of Agent $\mathrm{A}$ in that role) in a multi-agent system. The global reputation value of an agent (say A) in a multiagent system is determined as a weighted aggregate of its reputation in each of the three roles.

Carter et al $[10,11]$ propose two linguistic trust levels namely that an agent can have - trustworthy and 
untrustworthy. Based on fuzzy rules and the computed reputation value of an agent, the membership degree to which an agent can be classified as trustworthy or untrustworthy is determined.

They propose an approach along similar lines as the one discussed above for an information sharing society [12]. The roles identified by them that can be enacted by an agent (say A) in an information sharing society are as a social information provider (providing honest and accurate referrals to other agents), interactivity role (contributing information to other agents), content provider role (contributing high quality knowledge that is useful to other agents), administrative feedback role (providing feedback about the different aspects of the information sharing system like speed of the system, stability of the system, ease-of-use of the system and the quality of information available in the system) and the longevity role (whether an agent contributes positively to the multi- agent system). The reputation value that an agent (say A) can have in an information sharing society is determined as a weighted aggregate of its reputation in each of the five roles.

Carter et al [12] propose two linguistic trust levels that an agent can have - trustworthy and untrustworthy. Based on fuzzy rules and the computed reputation value of an agent, the membership degree to which an agent can be classified as trustworthy or untrustworthy can be determined.

\section{Fuzzy Logic Based Approach to trust and reputation computation proposed by Ramchurn et al}

Ramchurn et al [13,14] propose a fuzzy logic method which is based on FIRE, proposed by Huynh et al [15-18]. Ramchurn et al [13,14] propose three levels for expressing trust, as a result of direct interaction, which expressed in linguistic terms are Bad, Average and Good. Numerically they correspond to $-1,0$ and 1 respectively.

Based on the computed trust level, Agent A makes use of fuzzy logic rules to determine the membership degree for each of the three linguistic trust levels (or the confidence for the particular level of trust). Following other researchers, they propose that each agent (Agent A) would fix its own threshold on the number of interactions needed to determine the trust value of another agent (Agent B) accurately. When making a trust-based decision regarding interaction with Agent B, if the number of times Agent A interacted with Agent B is greater than or equal to the specified threshold, then Agent A makes use of the trust value obtained as a result of direct interaction between itself and Agent B to make a trust-based decision regarding interaction. However, if the number of times when Agent A has interacted with Agent B is less than the specified threshold, then Agent A would solicit recommendations about Agent B from other agents. In case Agent $\mathrm{A}$ solicits recommendations about Agent B, then:

1. The other agents (assume Agent C) communicate to Agent $A$ the membership degrees or the confidence values that they have in each of the three linguistic levels of trust for Agent B.

2. Agent $A$ then determines the reputation of Agent $B$ in each reputation level, by aggregating all the confidence values corresponding to each of the three linguistic levels individually.

3. Agent $\mathrm{A}$ then determines the trust level for Agent $\mathrm{B}$ in a given linguistic trust level by computing a weighted aggregate of the confidence that Agent A has in Agent B (based on direct interaction between them) for that linguistic trust level and the reputation of Agent B for that particular linguistic trust level.

\section{Conclusion}

In this paper we presented an overview of the existing soft-computing based approaches for trust and reputation computation. In Section 2, we discussed ReGreT, which is a soft-computing based method proposed by Sabater et al. In Section 3, we discussed the fuzzy-logic based approach to trust and reputation computation proposed by Shanshan Song et al. In Section 4, we discussed the neural network based approach to trust and reputation computation proposed by Weihua Song et al. In Section 5, we discussed the fuzzy-logic based approach to trust and reputation computation proposed by Carter and Ghorbani et al. Finally, in Section 6, we discussed the fuzzy-logic based approach to trust and reputation computation proposed by Ramchurn et al.

\section{References}

[1] Jordi Sabater, Carles Sierra, "REGRET: reputation in gregarious societies", Proceedings of the $5^{\text {th }}$ International Conference on Autonomous Agents (Agents 2001), Montreal, Canada, May 28 - June 1 2001, pp.194-195. 
[2] Jordi Sabater, Carles Sierra, "Reputation and social network analysis in multi-agent systems", Proceedings of the First International Joint Conference on Autonomous Agents and Multi-Agent System (AAMAS 2002), Bologna, Italy, 15-19 July 2002, pp. 475-482.

[3] Jordi Sabater, "Evaluating the Regret System", Applied Artificial Intelligence, Volume 18, Issue (9-10), UK, October-December 2004, pp.797-813.

[4] Shanshan Song, Kai Hwang, Runfang Zhou, Yu-Kwong Kwok, "Trusted P2P Transactions with Fuzzy Reputation Aggregation", IEEE Internet Computing, Volume 9, Issue 6, USA, November-December 2005, pp. 24-34.

[5] Shanshan Song, Kai Hwang, Mikin Macwan, "Fuzzy Trust Integration for Security Enforcement in Grid Computing", Proceedings of the IFIP International Conference on Network and Parallel Computing (NPC 2004), Wuhan, China, 18-20 October 2004, pp. 9-21.

[6] Kai Hwang, Yu-Kwong Kwok, Shanshan Song, Min Cai Yu Chen, Ying Chen, Runfang Zhou, Xiaosong Lou, "GridSec: Trusted Grid Computing with Security Binding and Self-defense Against Network Worms and DDoS Attacks", Proceedings of the $5^{\text {th }}$ International Conference on Computational Science (ICCS 2005), Atlanta, USA, 22-25 May 2005, 187-195.

[7] Weihua Song, Vir V. Phoha, "Neural Network-Based Reputation Model in a Distributed System", Proceedings of the 2004 IEEE International Conference on E-Commerce Technology (CEC 2004), San Diego, USA, 6-9 July 2004, pp.321-324.

[8] Weihua Song, Vir V. Phoha, "Opinion Filtered Recommendation Trust Model in Peer-to-Peer Networks", Proceedings of the $2^{\text {nd }}$ International Workshop on Agents and Peer to Peer Computing (AP2PC 2004), Melbourne, Australia, 14 July 2003, pp. 237-244.

[9] Giorgos Zacharia, Alexandros Moukas, Pattie Maes, "Collaborative Reputation Mechanisms in Electronic Marketplaces", Decision Support Systems, Volume 29, Issue 4, December 2000, Elsevier Science, pp. 371-388.

[10] Jonathan Carter, Ali A. Ghorbani, "Towards a Formalization of Trust", Web Intelligence and Agent Systems, Volume 2, Issue 3, IOS Press, pp. 167-183.
[11] Jonathan Carter, Ali A. Ghorbani, "Value Centric Trust in Multi-Agent Systems", Proceedings of the 2003 IEEE/WIC International Conference on Web Intelligence (WI 2003), Halifax , Canada, 13-16 October 2003, pp. 3-9.

[12] Jonathan Carter, Elijah Bitting, Ali A. Ghorbani, "Reputation Formalization for an Information-Sharing Multi-Agent System", Computational Intelligence, Volume 18, Issue 4, USA, pp. 515-534.

[13] Sarvapali D. Ramchurn, Nicholas R. Jennings, Carles Sierra, Lluis Godo, "Devising A Trust Model For MultiAgent Interactions Using Confidence And Reputation", Applied Artificial Intelligence, Volume 18, Issue 9-10, October- December 2004, Taylor and Francis Publishers, USA, pp. 833-852.

[14] Sarvapali D. Ramchurn, Carles Sierra, Lluis Godo and Nicholas R. Jennings, "A computational trust model for multi-agent interactions based on confidence and reputation", Proceedings of the $6^{\text {th }}$ International Workshop of Deception, Fraud and Trust in Agent Societies, Melbourne, Australia, 14-15 July 2003, pp. 69-75.

[15] Trung Dong Huynh, Nicholas R. Jennings, Nigel R. Shadbolt, "FIRE: An Integrated Trust and Reputation Model for Open Multi-Agent Systems", Proceedings of the $16^{\text {th }}$ European Conference on Artificial Intelligence (ECAI 2004), Valencia, Spain, 22-27 August 2004, pp. $18-22$.

[16] Trung Dong Huynh, Nicholas R. Jennings, Nigel R. Shadbolt, "Developing an integrated trust and reputation model for open multi-agent systems". Proceedings of the $7^{\text {th }}$ International Workshop on Trust in Agent Societies, New York, USA, 19 July 2004, 6574.

[17] Trung Dong Huynh, Nicholas R. Jennings, Nigel R. Shadbolt, "On Handling Inaccurate Witness Reports", Proceedings of the $8^{\text {th }}$ International Workshop on Trust in Agent Societies, Utrecht, The Netherlands, July 25July 26 2005, pp.63-77.

[18] Trung Dong Huynh, Nicholas R. Jennings, Nigel R. Shadbolt, "An Integrated Trust and Reputation Model for Open Multi-Agent Systems". Journal of Autonomous Agents and Multi-Agent Systems, Springer Verlag, The Netherlands (To Appear, 2006). 